subsequent studies identified complex of clinical, laboratory and morphological factors contributing to development of trajectories. Structural OA variants are diverse depending on visualization methods, and many of them can be naturally considered phenotypes, since they drive certain clinical OA manifestations. Morphological changes were described at macro- and microscopic levels; it is interesting to note the absence of histopathological norm in patients without OA. Laboratory profiles of patients are determined by content of systemic (serum, urinary) or local, "proximal" (in synovial fluid) biomarkers, which seem to be more precise. Metabolomic analysis is perspective new direction of laboratory studies based on joint metabolic products identification in the synovial fluid. New trend in OA research is molecular phenotyping. The specific molecular pathway explaining observed phenotype properties is called "endotype." Endotype is related to certain pathobiological scenario, and laboratory markers are potentially effective for its diagnosis.

Conclusion: Thus, a large amount of accumulated information and its diversity soon will probably lead to qualitatively new knowledge level with deep understanding of phenotype-associated strategy for managing OA patients.

REFERENCES:

[1] Wallace IJ, Worthington S, Felson DT, et al. Knee osteoarthritis has doubled in prevalence since the mid-20th century. Proc Natl Acad Sci USA. 2017 Aug 29;114(35): 9332-9336. doi: 10.1073/pnas.1703856114 Epub 2017 Aug 14.

[2] Deveza LA, Nelson AE, Loeser RF. Phenotypes of osteoarthritis: current state and future implications. Clin Exp Rheumatol 37 Suppl 2019;120(5):64-72.

[3] Van Spil WE, Bierma-Zeinstra SMA, Deveza LA, et al. A consensus-based framework for conducting and reporting osteoarthritis phenotype research. Arthritis Res Ther. 2020;22(1):54. doi:10.1186/s13075-020-2143-0

Disclosure of Interests: None declared.

DOI: 10.1136/annrheumdis-2021-eular.36

\section{AB0583 1 FRAILTY IN THE PATIENTS WITH OSTEOARTHRITIS OF THE KNEE WAS NOT CAUSED BY SARCOPENIA}

K. Inui ${ }^{1}{ }^{, 2}$, K. Mandai ${ }^{1}$, T. Ikawa ${ }^{1}$, Y. Minami ${ }^{1}$, K. Ueno ${ }^{1}$, H. Ohashi ${ }^{1}{ }^{1}$ Osaka Saiseikai Nakatsu Hospital, Orthopaedic Surgery, Osaka, Japan; ${ }^{2}$ Osaka City University Graduate School of Medicine, Orthopaedic Surgery, Osaka, Japan

Background: Osteoarthritis of the knee (knee OA) decreases mobility of the elderly, which function could be significantly improved with artificial joint replacement in many cases. Successful results of the surgery depend on several factors including preoperative muscle strength of lower limbs.

Objectives: We assessed morbidity and skeletal muscle mass and strength in patients with knee OA immediately before undergoing arthroplasty and investigated the relationship between impairment of mobility and skeletal muscle function.

Methods: All patients scheduled to undergo knee arthroplasty at our hospital after July 2020 were assessed for basic attributes, clinical assessment, blood tests, radiography, whole-body mode DXA, knee muscle strength by dynamometer with written consent (UMIN ID: 000040940). And Japanese Cardiovascular Health Study criteria for frailty, and sarcopenia by Asian Working Group for Sarcopenia 2019 criteria were evaluated.

Results: Among 46 patients (40 women, mean age 75.4 years) the overall distribution in frailty is no: $15.9 \%$, pre-frailty: $56.8 \%$, and frailty: $27.3 \%$. That in sarcopenia is no: $91.3 \%$, yes: $2.2 \%$, and severe: $4.3 \%$. Ninety-one $\%$ of the patients with frailty did not suffer from sarcopenia. Between the patients with frailty and those without frailty there was a significant difference in walking speed $(0.71 \pm 0.29 \mathrm{~m} /$ sec, $1.12 \pm 0.29 \mathrm{~m} / \mathrm{sec} ; \mathrm{p}=0.004$ adjusted by age and sex). Meanwhile, between those 2 groups of patients no significant differences were observed in skeletal muscle mass $\left(6.40 \pm 0.87 \mathrm{~kg} / \mathrm{m}^{2}, 6.59 \pm 1.09 \mathrm{~kg} / \mathrm{m}^{2} ; \mathrm{p}=0.35\right)$ and knee extension power $(120.4 \pm 37.4 \mathrm{~N}, 143.7 \pm 67.9 \mathrm{~N} ; \mathrm{p}=0.88)$ adjusted by age and sex. Together with these results, decrease in lower limb motor functions of the patients with knee OA was not caused by sarcopenia. In other words, improvement of mobility in the patients with knee OA could be expected by surgical intervention because of pain relief and ROM improvement.

Conclusion: In the patients with knee OA immediately before arthroplasty, frailty was not caused by sarcopenia.

Disclosure of Interests: None declared.

DOI: 10.1136/annrheumdis-2021-eular.416

\section{AB0584 \\ THE QUALITY OF REPORTING IN RANDOMIZED CONTROLLED TRIALS OF HOME BASED REHABILITATION FOR KNEE OSTEOARTHRITIS: UTILIZING PEDRO SCORE AND CONSORT CRITERIA}

M. Meran ${ }^{1,2}$, B. Unver ${ }^{1}$, E. Çağlar ${ }^{3} .{ }^{1}$ Dokuz Eylul University, School of Physical Therapy and Rehabilitation, Izmir, Turkey; ${ }^{2}$ Istanbul Beykoz University, School of Physical Therapy and Rehabilitation, Istanbul, Turkey; ${ }^{3}$ Istanbul Gelisim University, Faculty of Health Science/ Physical Therapy and Rehabilitation, Istanbul, Turkey
Background: Osteoarthritis of the knee (KOA) is a very common rheumatic disease, and its global burden is gradually increasing (1). The benefits of exercise in patients with KOA are supported by high-level evidence and take their place in primary care therapy (2). Today, coronavirus disease 2019 has developed as a pandemic all over the world, creating difficulties in healthcare and highlighting home-based rehabilitation ( $\mathrm{HBR}$ ) (3). Randomized controlled trials (RCTs) are considered the gold standard for evaluating the effects of clinical interventions but poorly reported results can have negative consequences. The Physiotherapy Evidence Database (PEDro) evaluates the methodological quality of RCTs (4). The CONSORT (Consolidated Standards of Reporting Trials) statement has been developed to improve the reporting quality of RCTs (5). There are no studies examining the quality of RCTs related to HBR in patients with KOA.

Objectives: The aim of this study was to assess the reporting quality of HBR trials for KOA, and explore the factors associated with the reporting.

Methods: Two independent researchers investigated HBR RCTs in patients with KOA published between 1999 and 2020 were sourced from PubMed, the Cochrane Reviews and Web of Science. Each researcher evaluated the methodological quality of the included studies using the PEDro scoring and reporting aspects using 9 items from CONSORT. The relationship between adherence to the CONSORT criteria and the PEDro score were evaluated.

Results: Twenty-five RCTs met our eligibility criteria. The mean PEDro score of studies is $5.76 \pm 1.48$. Only one study found high quality (PEDro score: $\geq 9$ ). The PEDro scores were: randomization type $(96 \%, 24 / 25)$ and baseline comparability $(92 \%, 23 / 25)$; all studies described group comparisons and variablity measures. In contrast, concealed allocation (18\%, $7 / 25)$, blinding of participants (16\%, 4/25) and therapists $(0 \%)$ were not included in most studies. The CONSORT criteria were: flow diagram $(92 \%, 23 / 25)$, sample size, subgroup analysis and sources of funding $(56 \%, 11 / 25$ for all three), while the majority of studies the trial registration number $(16 \%, 4 / 25)$ was not available. A high level of correlation was found between meeting the CONSORT criteria and PEDro scores $(r=0.820, p<0.001)$. Conclusion: The majority of RCTs based on HBR for KOA are low-to-modarate quality studies based on the PEDro score. Adherence to the CONSORT criteria is linked to high quality scores. If the studies are planned and written in accordance with the CONSORT criteria, we think that better quality studies will emerge. REFERENCES:

[1] Safiri S, Kolahi AA, Smith E, Hill C, Bettampadi D, Mansournia MA, et al. Global, regional and national burden of osteoarthritis 1990-2017: a systematic analysis of the Global Burden of Disease Study 2017. Ann Rheum Dis. 2020;79(6):819-28.

[2] Skou ST, Pedersen BK, Abbott JH, Patterson B, Barton C. Physical Activity and Exercise Therapy Benefit More Than Just Symptoms and Impairments in People With Hip and Knee Osteoarthritis. J Orthop Sport Phys. 2018;48(6):439-47.

[3] Karasavvidis T, Hirschmann MT, Kort NP, Terzidis I, Totlis T. Home-based management of knee osteoarthritis during COVID-19 pandemic: literature review and evidence-based recommendations. J Exp Orthop. 2020;7(1):52.

[4] Sherrington C, Herbert RD, Maher CG, Moseley AM. PEDro. A database of randomized trials and systematic reviews in physiotherapy. Man Ther 2000;5(4):223-6

[5] Moher D, Hopewell S, Schulz KF, Montori V, Gøtzsche PC, Devereaux PJ, et al. CONSORT 2010 explanation and elaboration: updated guidelines for reporting parallel group randomised trials. Int J Surg. 2012;10(1):28-55.

Disclosure of Interests: None declared.

DOI: 10.1136/annrheumdis-2021-eular.476

\section{AB0585 $\quad$ COMPARISON OF THE EFFECTIVENESS OF HYALURONIC ACID PREPARATIONS WITH DIFFERENT MOLECULAR WEIGHTS AND IN COMBINATION WITH CHONDROITIN SULFATE DEPENDING ON THE STAGE OF OSTEOARTHRITIS OF THE KNEE JOINT}

V. Bialik ${ }^{1}$, M. Makarov ${ }^{1}$, E. Bialik ${ }^{1}$, S. Makarov ${ }^{1}$, V. Nesterenko ${ }^{1}$,

M. Nurmukhametov ${ }^{1}{ }^{1}$ V. A. Nasonova Research Institute of Rheumatology,

Rheumoorthopedic and Rehabilitation, Moscow, Russian Federation

Background: intra-articular $(\mathrm{IA})$ injections of hyaluronic acid $(\mathrm{HA})$ preparations are one of conservative treatment of the knee osteoarthritis (OA). However, the results of this treatment are inconsistent.

Objectives: to evaluate the effectiveness of HA preparations with different molecular weights and in combination with chondroitin sulfate (HS) for IA injections in the treatment of patients with stage I-III knee OA.

Methods: IA HA injections were performed 160 patients with primary and post-traumatic knee OA of the I-III stages at the department of rheumoorthopaedic and rehabilitation, V. A. Nasonova Research Institute of Rheumatology for the period from September 2017 to June 2019. Patients were divided into 4 groups. Group 1 consisted of 80 patients treated with low molecular weight (LMW) HA group 2 - 20 patients treated with medium molecular weight (MMW) HA, group 3 - 30 patients treated with high molecular weight (HMW) HA, and group $4-30$ 